\title{
APLICAÇÃO DE ATIVIDADE DE APRENDIZAGEM BASEADA EM PROBLEMA EM ENGENHARIA CIVIL: EXPERIÊNCIA NA DISCIPLINA DE TEORIA DAS ESTRUTURAS
}

DOI: 10.37702/2175-957X.COBENGE.2021.3626

ELIEDSON RAFAEL DE CARVALHO - eliedson.carvalho@ifal.edu.br Instituto Federal de Alagoas Av. Alagoas - Palmeira de Fora s/n 57608-180 - Palmeira dos Índios - AL

Álvaro Mello Lima - aml4@aluno.ifal.edu.br Instituto Federal de Alagoas

Rua Manoel Lúcio da Silva 700

57304-350 - Arapiraca - AL

João Pedro Soares Neto - jpsn1@aluno.ifal.edu.br Instituto Federal de Alagoas

Povoado Bonito 00

57635-000 - TANQUE D'ARCA - AL

George Lucas dos Santos - George.lucas.dss@gmail.com Instituto Federal de Alagoas

Rua Antônio Bernardino de sena 135

57306-250 - Arapiraca - AL

Everton Alberto Barbosa Ferreira - eabf1@aluno.ifal.edu.br Instituto Federal de Alagoas

Rua Sebastião Barbosa 169

57603-040 - Palmeira dos Índios - AL

Resumo: Este artigo tem como objetivo exibir os resultados obtidos na atividade aplicada na disciplina de Teoria das Estruturas II do curso de Engenharia Civil do Instituto Federal de Alagoas - IFAL, com base nas concepções de Aprendizagem Baseada em Problema - ABP como estratégia metodológica durante as aulas no período de ensino remoto emergencial. Em sua integridade, a organização da atividade se dividiu em três estágios: materiais e equipamentos, modelagem do 
problema e análise das reações. A atividade consistiu em analisar uma estrutura real realizando a observação de seus elementos, peso próprio e dimensões. Consequentemente, os estudantes foram designados a modelar a estrutura, elaborando o seu arranjo representativo com os devidos componentes em cada local específico. Desta maneira, os discentes utilizaram conceitos de cálculo estrutural abordados na disciplina de teoria das estruturas II para a obtenção das reações de apoio do arquétipo por meio de dois métodos distintos: Método dos Deslocamentos e Processo de Cross. Em seguida, o mesmo modelo foi analisado, para fins de comparação, no Ftool, software de análise estrutural de protótipos de projetos executivos profissionais. Por fim, pode ser afirmado que a análise das respostas da atividade de ABP proporcionou resultados satisfatórios, visto que os discentes resolveram a situação problema proposta, analisando a estrutura real, realizando os cálculos e desenvolvendo o comparativo com o software, através do processo prático de ensino-aprendizagem do objeto de estudo.

Palavras-chave: Aprendizagem baseada em problema. Engenharia civil. Teoria das estruturas. 


\section{APLICAÇÃO DE ATIVIDADE DE APRENDIZAGEM BASEADA EM PROBLEMA EM ENGENHARIA CIVIL: EXPERIÊNCIA NA DISCIPLINA DE TEORIA DAS ESTRUTURAS}

\section{INTRODUÇÃO}

O sistema de aprendizado e educação têm sido um assunto de grandes contestações, seja em qualquer área. Formas de ensino vêm sendo analisadas e discussões acontecem sob diversos panoramas que guiam as práticas pedagógicas incorporada. Sabe-se que o intuito é manter o sistema de aprendizagem expressivo e relevante, e que os profissionais envolvidos nas áreas da ciência precisam articular e apresentar processos que facilitem e melhorem as relações de estudo.

A respeito das maneiras de aprendizado, Pereira et al. (2007) inferem que a limitação da tradicional proposta, aliada a inovações crescentes, impulsionaram a comunidade científica, a pesquisar alternativas de novos processos de ensino-aprendizagem, capazes de formar os futuros engenheiros com visão holística, integrando a ciência com a prática, através do aprendizado ativo e baseado em competências.

Hoje em dia, no âmbito da Engenharia Civil, o ato de ensinar e aprender envolve algumas peculiaridades, pois, dentro deste processo a mediação entre os objetos de estudo, aluno, professor e o sistema de aprendizado, estão diretamente atrelados à prática, exigindo atividades mútuas e participativas. Dentro desta argúcia nota-se que deve existir um envolvimento ideal entre todas as partes, abrindo-se espaço para a aplicação de atividades práticas, de bom desempenho, validando todo o conhecimento a ser transmitido.

Desta maneira, de acordo com Moreiras et al. (2021) o desenvolvimento do raciocínio lógico e crítico para a solução de problemas é adverso ao ser passado em salas de aula e web conferências, visto que isso prejudica a formação do aluno na capacidade de solucionar problemas de Engenharia do dia a dia.

Portanto, deve-se motivar o aluno e treiná-lo para resolver problemas específicos relativos aos conteúdos transmitidos em sala de aula com outras ferramentas e métodos específicos,

Entre as metodologias, para este contexto, cita-se a Atividade Baseada em Problema - ABP (Problem Based Learning - PBL), que propicia a solução de problemas durante as orientações na disciplina estudada através da tutoria do professor. Desta forma, o aluno passa a ser instrumento de aprendizagem, e o ensino torna-se significativo dentro da abordagem dos assuntos, envolvendo a prática e os conceitos abordados.

Por fim, este artigo tem como objetivo apresentar os resultados obtidos na Atividade Baseada em Problema proposta para a disciplina de Teoria das Estruturas II, como alternativa metodológica durante o ensino remoto emergencial, no período de 2020.2.

\section{MATERIAIS E MÉTODOS}

Objetivando trabalhar com os métodos e procedimentos instruídos por uma metodologia de aprendizagem baseada em Problema (ABP) desenvolvida na disciplina de Teoria das Estruturas II do Instituto Federal de Alagoas - IFAL - Campus Palmeira dos Índios, o presente trabalho ostenta um método de desenvolvimento quantitativo e descritivo, se baseando em análises de informações e cálculo de um modelo estrutural.

Desta maneira, a metodologia deste trabalho pode ser dividida em três etapas: materiais e equipamentos, modelagem do problema e análise das reações. 


\subsection{Materiais e equipamentos}

A estrutura designada para a realização do trabalho consistiu em uma placa cimentícia de $10 \mathrm{~mm}$ de espessura, bi engastada em um perfil de alumínio por meio de parafusos, e com suportes de cabo de arame galvanizado a cada metro de largura, sendo 4 metros de largura, portanto, 3 suportes. Em seu meio externo a placa foi revestida com massa base de tratamento de superfícies e porcelanato de dimensões $200 \times 1200 \times 10 \mathrm{~mm}$. $\mathrm{Na}$ Figura 1 é possível observar um dos ganchos, que funciona como apoio no sistema, um dos engastes a uma distância de 1 metro do gancho e um trecho da placa.

Figura 1 - Configuração de suporte e apoio da placa.

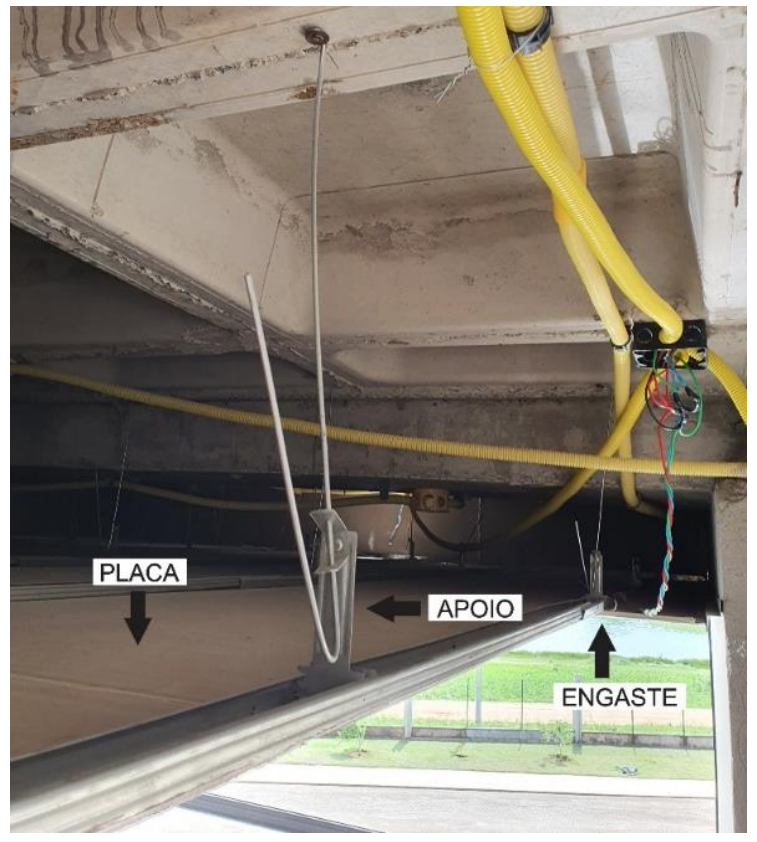

Fonte: Autores (2021)

A placa cimentícia corresponde a uma chapa de superfície polida, composta por liga cimentícia, fibra de vidro, perlita e componentes. Em sua maioria, as placas são utilizadas para soluções em sistemas de forros externos ou internos, beirais, shafts, platibandas, construções em light steel frame, wood frame, e outros, sendo uma alternativa de rápida aplicação e de boa resistência.

Por conseguinte, o porcelanato do revestimento, observado na Figura 2, que é indicado para aplicação em áreas externas sem coberturas, churrasqueiras, garagens, quintais e etc, possui formato retangular, textura lisa, e devido à sua borda reta retificada, foi aplicado com juntas menores em relação às lajotas, com um espaçamento de $2 \mathrm{~mm}$. $O$ estudo deste modelo foi usualmente expressado em termos de suas dimensões e intervalos, para que pudessem servir como valores de variáveis dependentes em sua análise, contudo, para a finalidade de tratamento do peso específico do porcelanato, foi utilizada a ABNT NBR 6120 (ABNT, 2019). 
Figura 2 - Aplicação do revestimento em massa e do porcelanato.

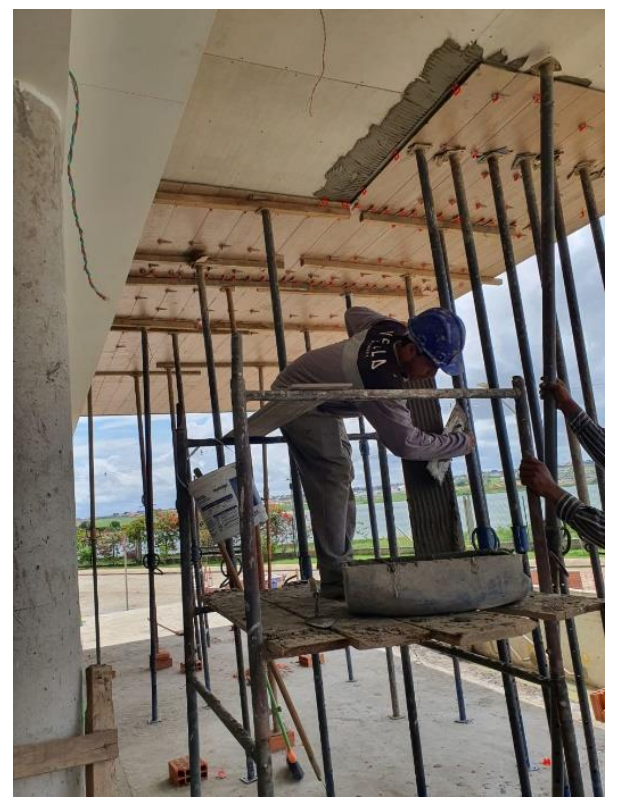

Fonte: Autores (2021)

\subsection{Modelagem do problema}

A configuração da placa estudada remete a um modelo geométrico estrutural capaz de suportar carregamentos lineares e esforços admissíveis em relação ao seu comprimento. Esta hipótese se torna válida pelo fato do comportamento dos elementos que compõem o perfil possuírem peso próprio e condições de exposição de cargas que exigem equilíbrio de referência em relação aos seus apoios, que são os ganchos.

Através da estrutura, criou-se um arranjo representativo geral em vista, com a finalidade de observar o posicionamento das peças que formam a estrutura, bem como suas dimensões, observado na Figura 3.

Figura 3 - Sistema representativo geral.

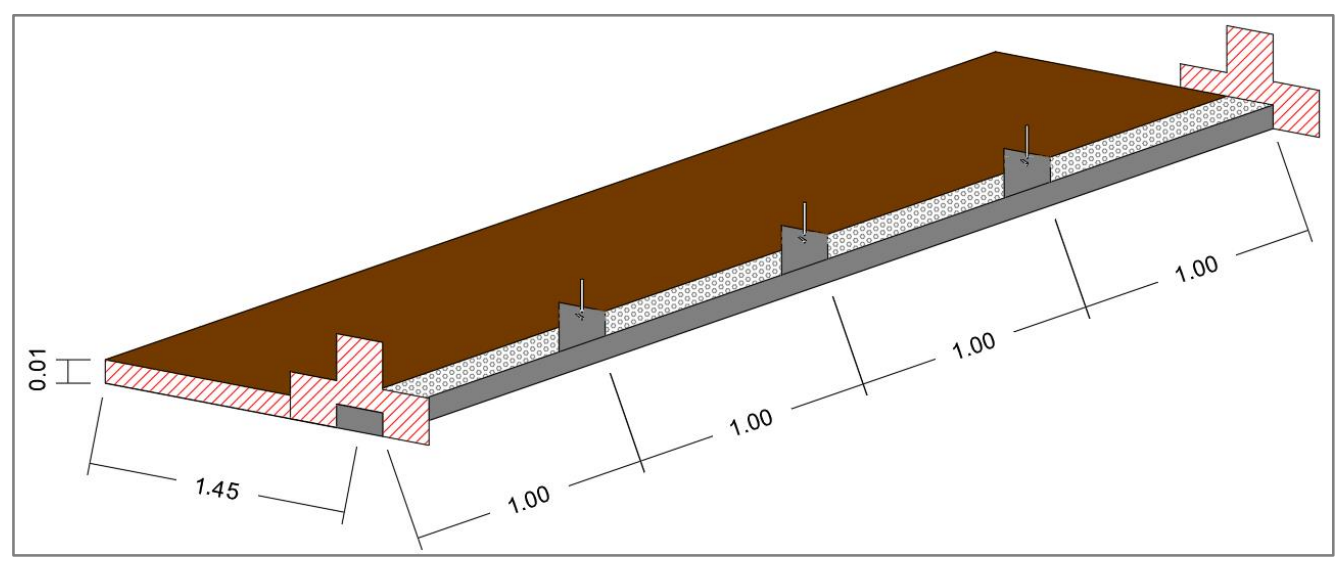

Fonte: Autores (2021) 
Para a modelização da estrutura, observa-se na Figura 4 que os ganchos sofrem uma reação no sentido vertical ascendente de forma a suportar a toda a massa do conjunto placa-revestimento, porém, não estando travado na direção do seu eixo horizontal, sendo esta uma função dos engastes nas extremidades.

Figura 4 - Detalhe da esquematização dos suportes da placa.

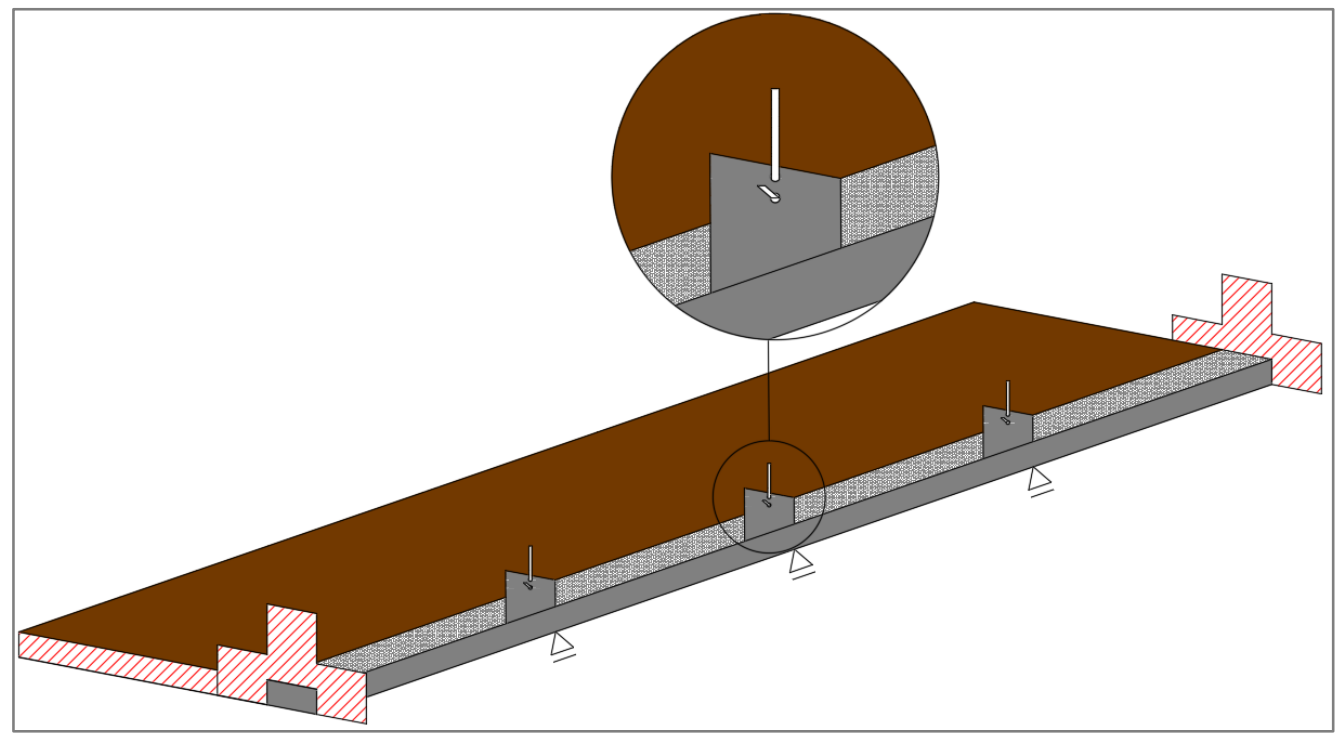

Fonte: Autores (2021)

Por conseguinte, através destas perspectivas, desenhou-se na Figura 50 arranjo sistemático genérico da estrutura para fins de cálculo e análises.

Figura 5 - Arranjo estrutural sistemático genérico.

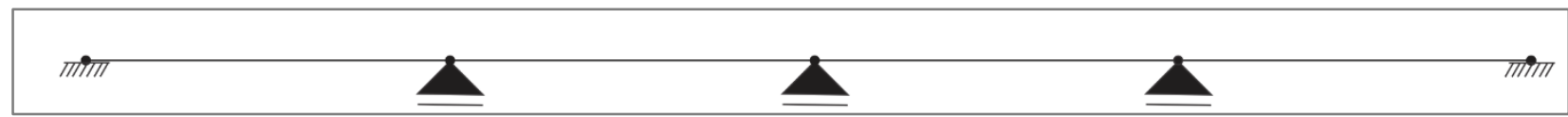

Fonte: Autores (2021)

O modelo de carregamento do sistema foi calculado se utilizando do peso próprio de cada item da estrutura, levando em conta uma fração de 1,45 metros de comprimento por 4 metros de largura que reúne uma das placas, incluindo sua estrutura, suporte e revestimento.

Desta maneira, os valores das ações em questão foram verificados caso a caso e consequentemente calculados entre si por meio da Equação 1, observada na NBR 6120.

$$
Q=G o \times L \times h \times V
$$

Em que $G o=$ Peso; $L=$ comprimento; $h=$ espessura; $V=$ vão.

\subsection{Análise das Reações}

As metodologias de cálculo das reações consistiram na utilização do Método dos Deslocamentos e do Processo de Cross. 
De acordo com Sussekind (1974) o método de Cross consiste num algoritmo interativo de grande simplicidade e rapidez para a resolução de estruturas. De maneira geral, acumula-se esforços de momento fletor em uma barra genérica da estrutura, ou seja, uma carga momento aplicada num nó de uma estrutura totalmente indeslocável irá se distribuir entre as diversas barras concorrentes neste nó, segundo parcelas proporcionais à rigidez.

Na Figura 6 constata-se um sistema estrutural, com as barras 1, 2 e 3. Já na Figura 7 , nota-se o seu sistema principal, com um sistema de engastamento perfeito no nó $A$, o qual insere-se rotações, funcionando como as cargas-momento. Através disto, resolve-se a estrutura sem utilizar equações de compatibilidade estática.

Figura 6 - Modelo de estrutura convencional.

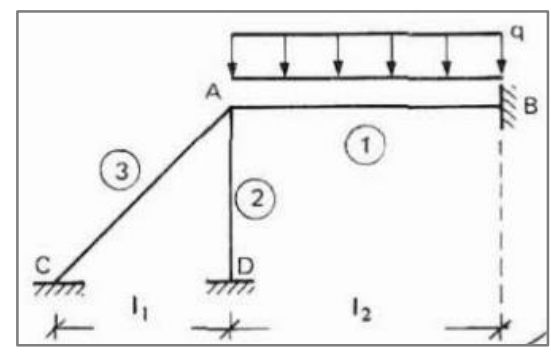

Fonte: Sussekind (1974)

Figura 7 - Engastamento perfeito no sistema principal.

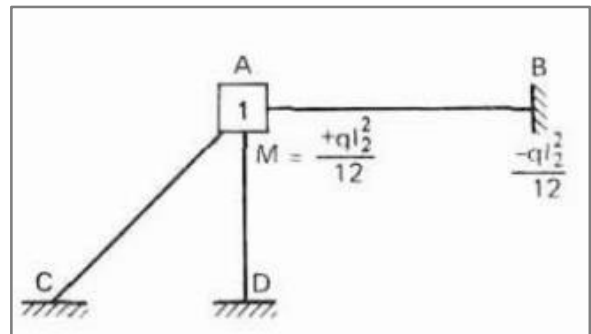

Fonte: Sussekind (1974)

Já o método dos deslocamentos consiste na aplicação de configurações deformadas conhecidas sobre a estrutura, associando-se cada uma a um determinado efeito, o qual é isolado. Conforme afirma Soriano (2004), escolhe-se um sistema principal com graus de liberdade restringidos, calcula-se os esforços de engastamento perfeito e coeficientes de rigidez das barras, para então resolver o sistema de equações de equilíbrio para determinação dos esforços. Na Figura 8 há um sistema com seus carregamentos e apoios.

Figura 8 - Viga representativa.

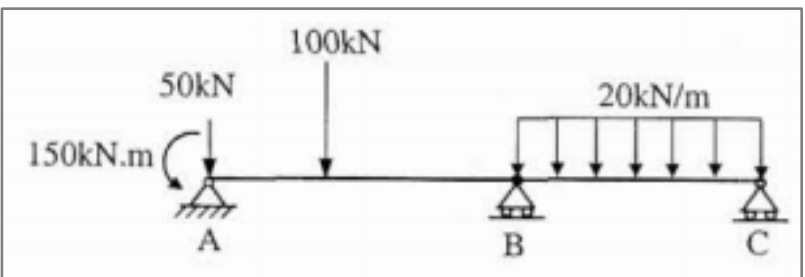

Fonte: Soriano (2004) 

apoio B.

Já na Figura 9, observa-se seu sistema principal com uma rotação d1 aplicada no

Figura 9 - Sistema principal com rotação d1 no engaste perfeito em $B$.

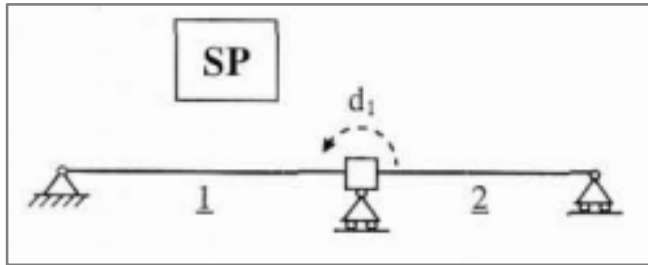

Fonte: Soriano (2004)

Nesta situação, d1 seria a primeira incógnita do sistema, e através desta, calcula-se os valores de engastamento perfeito e por conseguinte determina-se os demais esforços.

Portanto, através dessas metodologias e modelos baseou-se nas soluções para fins de interpretação, e realizou-se o comparativo com os resultados do software de análise estrutural Ftool.

\section{RESULTADOS E DISCUSSÃO}

Para as ações referentes ao porcelanato, conforme a ABNT NBR 6120, utilizou-se o peso específico $\mathrm{y}=23 \mathrm{kN} / \mathrm{m}^{3}$, já para a placa, conforme esclarecimentos do fabricante, utilizou-se o peso de $11,12 \mathrm{~kg} / \mathrm{m}^{2}$.

Como o Porcelanato possui uma espessura de $0,01 \mathrm{~m}$, obteve-se o peso de $0,23 \mathrm{kN} / \mathrm{m}^{2}$ por meio da sua espessura de revestimento nominal. Logo, observa-se na Tabela 1 o peso para cada componente da estrutura.

Tabela 1 - Peso dos componentes construtivos

\begin{tabular}{cc}
\hline Elemento & Peso (Go) \\
\hline Placa Cimentícia & $0,10909 \mathrm{kN} / \mathrm{m}^{2}$ \\
\hline Porcelanato & $0,23 \mathrm{kN} / \mathrm{m}^{2}$
\end{tabular}

Fonte: Autores (2021)

Portanto, possuindo o arranjo estrutural genérico e seus devidos pesos de atuação, utilizou-se a Equação 1 para a determinação do carregamento atuante ao longo do vão da estrutura, obtendo-se uma ação de 500N/m, como representado na Figura 10

Figura 10 - Modelo Efetivo de Cargas.

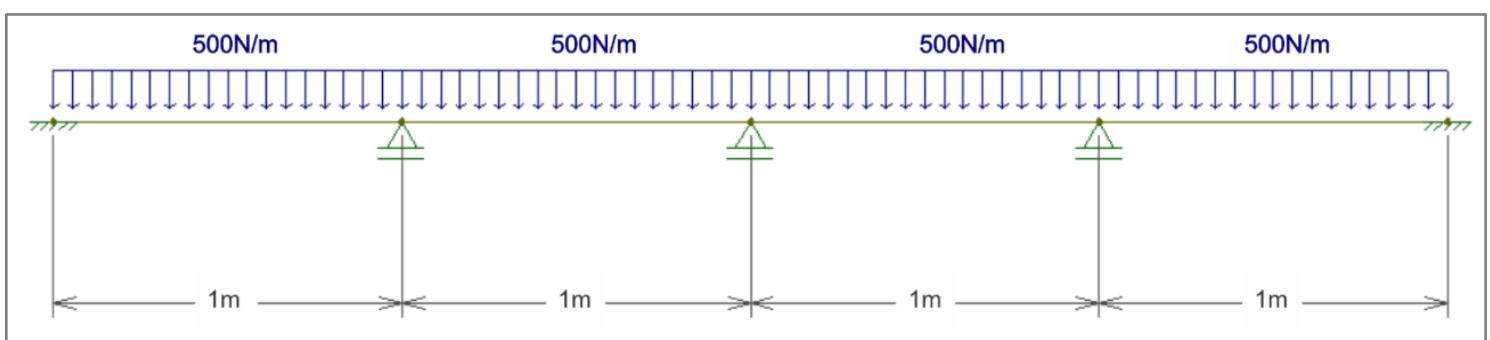

Fonte: Autores (2021)

Após a inserção do sistema estrutural genérico na ferramenta computacional Ftool, obteve-se os valores das reações atuantes observados na Figura 11. 
Figura 11 - Valores das reações atuantes ao longo da estrutura obtidos no Ftool.

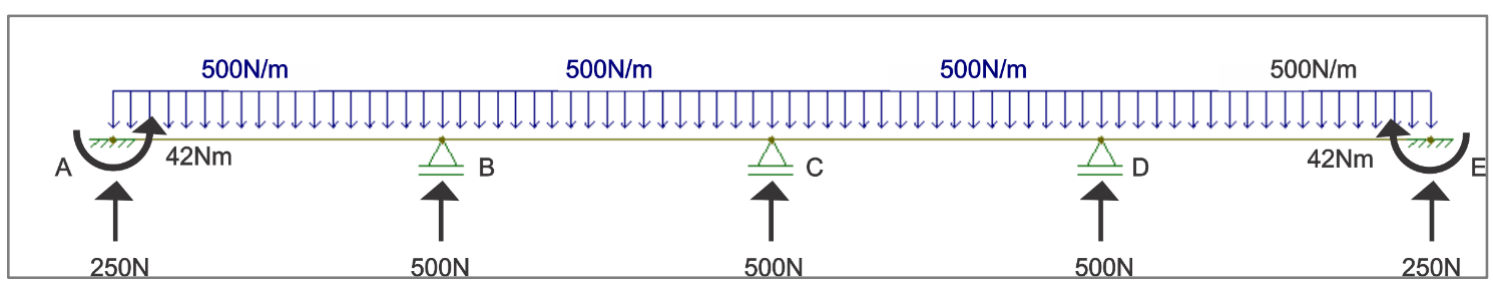

Fonte: Autores (2021)

Em seguida, foi realizado as análises através do Método de Cross e Método dos Deslocamentos

Por meio do Método de Cross, nos nós $A$ e $E$ obteve-se reação de apoio vertical de $250 \mathrm{~N}$ em ambos, momento fletor de $41,667 \mathrm{~N}$.m em A e de $-41,667 \mathrm{~N}$.m em E. Nos demais nós, chegou-se ao resultado de $500 \mathrm{~N}$ para as reações verticais, e momento fletor nulo.

Por conseguinte, através do Método dos Deslocamentos, calculou-se todas as reações verticais e de momento para 5 estados de atuação distintos, incluindo o sistema principal, através do quais se chegou aos valores de $250 \mathrm{~N}$ para as reações verticais nos nós $A$ e $E$, e de $500 \mathrm{~N}$ para as reações verticais em $B, C$ e $D$. Os momentos foram de $41,67 \mathrm{kN}$.m em A e $-41,67 \mathrm{kN} . \mathrm{m}$ em E. Assim sendo, temos na Tabela 2 os valores de reações obtidos por cada método estudado:

Tabela 2 - Resultados

\begin{tabular}{c|c|c|c|c|c|c}
\hline \multirow{2}{*}{ Método } & \multirow{2}{*}{ Reações } & \multicolumn{5}{|c|}{ Nó } \\
\cline { 3 - 7 } & & A & B & C & D & E \\
\hline \multirow{3}{*}{ Cross } & Vertical & $0,25 \mathrm{kN}$ & $0,5 \mathrm{kN}$ & $0,5 \mathrm{kN}$ & $0,5 \mathrm{kN}$ & $0,25 \mathrm{kN}$ \\
\cline { 2 - 7 } & Momento & $41,667 \mathrm{~N} \cdot \mathrm{m}$ & & & & - \\
& & & & & $41,667 \mathrm{~N} \cdot \mathrm{m}$ \\
\hline \multirow{2}{*}{ Deslocamentos } & Vertical & $0,25 \mathrm{kN}$ & $0,5 \mathrm{kN}$ & $0,5 \mathrm{kN}$ & $0,5 \mathrm{kN}$ & $0,25 \mathrm{kN}$ \\
\cline { 2 - 7 } & Momento & $41,67 \mathrm{~N} \cdot \mathrm{m}$ & & & & $-41,67 \mathrm{~N} \cdot \mathrm{m}$ \\
\hline \multirow{2}{*}{ Ftool } & Vertical & $0,25 \mathrm{kN}$ & $0,5 \mathrm{kN}$ & $0,5 \mathrm{kN}$ & $0,5 \mathrm{kN}$ & $0,25 \mathrm{kN}$ \\
\cline { 2 - 7 } & Momento & $42 \mathrm{kN} \cdot \mathrm{m}$ & & & & $-42 \mathrm{~N} \cdot \mathrm{m}$ \\
\hline
\end{tabular}

Fonte: Autores (2021)

Observa-se que as solicitações das reações de apoio na estrutura, através dos três métodos de cálculo distintos, apresentam variações sucintas na ordem da terceira casa decimal, e em alguns casos não apresentam variações. Logo, em função desta análise, nota-se que o comportamento das reações da placa estudada vistos através dos dois processos de cálculo manual, ou através do software, apresentam resultados relevantes e equivalentes entre si.

\section{CONSIDERAÇÕES FINAIS}

Diante dos pressupostos, conclui-se que foi possível elencar os resultados por meio dos diferentes métodos, como também do software Ftool, atingindo resultados satisfatórios, visto que as diferenças entre os valores são irrisórias. Considera-se que a estratégia da ABP proporcionou aos discentes uma visão diligente e compenetrada em relação aos conteúdos trabalhados. Evidencia-se que o método consistiu em uma iniciativa importante, pois, contribuiu para o refinamento do progresso relativo ao processo de aprendizagem diante de uma nova ocasião de ensino. 
Por fim, pode ser afirmado que a análise das respostas da atividade de $A B P$ proporcionou resultados satisfatórios, visto que os discentes resolveram a situação problema proposta, analisando a estrutura real, realizando os cálculos e desenvolvendo o comparativo com o software, através do processo prático de ensino-aprendizagem do objeto de estudo.

\section{REFERÊNCIAS}

ASSOCIAÇÃO BRASILEIRA DE NORMAS TÉCNICAS. NBR 6120: Ações para cálculo de estruturas de edificações. Rio de Janeiro, 2019.

FTOOL do Rio de Janeiro: banco de dados. Disponível em: https://www.ftool.com.br/Ftool/. Acesso em: 08 maio 2021.

MOREIRAS, Sérgio Trajano Franco et al. Aprendizagem baseada em problemas: disciplina de estruturas de concreto armado do curso de engenharia civil. Brazilian Journal of Development, v. 7, n. 4, p. 34401-34413, 2021.

PEREIRA, Clarisse Ferrão et al. Aprendizagem Baseada em Problemas (ABP)-Uma proposta inovadora para os cursos de engenharia. Simpósio de Engenharia de Produção-XIV SIMPEP 2007, 2007.

SORIANO, H. L.; LIMA, S. S.; Análise de Estruturas. v. 1. Rio de Janeiro: Editora Ciência Moderna LTDA, 2004.

SUSSEKIND, J. C.; Curso de Análise Estrutural. 7. ed. v. 3. Rio de Janeiro: Editora Globo, 1987. 


\title{
APPLICATION OF PROBLEM BASED LEARNING ACTIVITY IN CIVIL ENGINEERING: EXPERIENCE ON THE COURSE OF THEORY OF STRUCTURES
}

\begin{abstract}
This paper aims to show the results obtained in the activity applied in the course of Theory of Structures II of the Civil Engineering undergraduate program at the Federal Institute of Alagoas - IFAL, based on the conceptions of a Problem Based Learning - PBL as a methodological strategy in the classes during the period of emergency remote education. In general, the organization of the activity was divided into three stages: materials and equipment, problem modeling and analysis of support reactions. The activity consisted of analyzing a real structure, observing its elements, loads and dimensions. Consequently, the students were assigned to model the structure, elaborating its representative arrangement with the appropriate components in each specific location. In this way, the students used concepts of structural analysis approached in the course of theory of structures II to obtain the support reactions of the archetype through two different methods: Displacement Method and Cross Process (Moment Distribution Method). Then, the same model was analyzed, for comparison purposes, in Ftool, structural analysis software for prototypes of professional executive projects. Finally, it can be stated that the analysis of the answers of the activity of PBL provided satisfactory results, since the students solved the proposed problem situation, analyzing the real structure, performing the calculations and developing the comparison with the software, through the practical teaching-learning process of the study object.
\end{abstract}

Keywords: Problem based learning. Civil engineering. Theory of structures. 